\title{
Acción social y procesos organizativos en la Iglesia católica
}

\author{
Ángel Belzunegui, Ignasi Brunet \\ Universidad Rovira i Virgili \\ angel.belzunegui@urv.cat; ignasi.brunet@urv.cat
}

Resumen: Presentamos aquí los resultados de una investigación realizada el año 2008 y patrocinada por la Archidiócesis de Tarragona, que analizó la acción social de las entidades católicas. La hipótesis inicial fue que la cobertura de la acción social se produce como resultado del grado de descentralización de las entidades católicas. El diseño de la investigación combinó técnicas cualitativas y técnicas cuantitativas. El análisis de la información conduce a la conclusión de que las entidades religiosas forman un esquema organizativo en forma de constelación del que deriva precisamente su eficacia a la hora de desplegar la cobertura y la intensidad de la acción social.

Palabras clave: Organización, acción social, entidades sociales

Abstract: We present here the results of an investigation carried out in 2008 and sponsored by the archdiocese of Tarragona, which analyzes the social mission of Catholic institutions. The initial hypothesis was that the social mission depended on the degree of decentralization of the Catholic institutions. The research design combined qualitative and quantitative techniques. The analysis of the information led to the conclusion that religious institutions are organized in the form of a constellation, which is precisely the reason why they are so effective at complying with such an extensive and intense social mission.

Key words: Organization, social mission, social institutions 


\section{Introducción}

Como señalamos en la presentación de esta comunicación, las conclusiones que presentamos en este texto derivan del análisis realizado en el estudio «La Acción Social de la Iglesia», un estudio encargado por Cáritas Diocesana de Tarragona cuyo objetivo general fue la realización del mapa de la actividad social de la Iglesia en la Archidiócesis de Tarragona.

La hipótesis del estudio fue que, a mayor descentralización en la organización de la acción social de las entidades católicas, mayor alcance de éstas al conjunto social. Este hecho se consideró, en sí mismo, un indicador de la eficacia de la propia acción social; por consiguiente, en la hipótesis inicial vinculamos la descentralización y la autonomía de la acción con su grado de eficacia, teniendo en cuenta el número de personas atendidas y la implantación en el territorio de la acción.

El diseño metodológico del estudio combinó técnicas cualitativas (análisis documental y entrevistas en profundidad) con técnicas cuantitativas (encuesta a través de cuestionario). Para la realización de las entrevistas en profundidad se diseñó una estrategia de identificación de lo que podríamos llamar nódulos de la red o la matriz de entidades que llevan a cabo la acción social. En el espacio de relaciones entre las entidades observamos la centralidad de la figura de los arciprestes como figuras que desempeñan un papel de coordinación, por lo que procedimos a entrevistarlos.

Iniciamos el estudio con entrevistas en profundidad a los 11 arciprestes del territorio, a través de un guión de entrevista estructurado. El objetivo de estas entrevistas fue identificar las entidades católicas que realizan acción social en el territorio de su magisterio. Estas entrevistas sirvieron para situarnos en el contexto del objeto de estudio y para conocer de primera mano la filosofía de actuación que acompaña a la mayor parte de esta acción social. Su conocimiento de las personas y de la situación de las comunidades los convirtieron en unos informantes privilegiados.

Una vez identificadas las acciones sociales de la Iglesia en el territorio, comenzamos el trabajo de campo a través de encuestas a las diferentes entidades y a las personas que prestan asistencia social. Teniendo en cuenta la gran cantidad de entidades de la Iglesia en materia de bienestar social, nos propusimos entrevistar al mayor número posible de personas y entidades vinculadas a la acción social. La estrategia para la selección de las unidades de información fue intentar cubrir en su totalidad las comunidades religiosas y los centros sociales, y, por lo que respecta a las Cáritas parroquiales, recogimos la información, por un lado, de los registros centrales de Cáritas Diocesana, y, por otro, de una encuesta a una muestra de voluntarias y voluntarios de la entidad.

Los números del trabajo de campo del estudio han sido los siguientes: 
Tabla 1. El trabajo de campo en cifras

\begin{tabular}{|l|l|c|c|c|}
\hline \multicolumn{2}{|l|}{} & $N\left(^{*}\right)$ & $n\left(^{* *}\right)$ & $\%$ de $n / N$ \\
\hline & Entrevistas exploratorias al inicio del estudio & 5 & 5 & $100 \%$ \\
\hline & Entrevistas en profundidad a arciprestes & 11 & 10 & $91 \%$ \\
\hline & Entrevistas en profundidad a comunidades religiosas & 60 & 43 & $71,6 \%$ \\
\hline Entrevistas en profundidad con centros sociales & 12 & 10 & $83,3 \%$ \\
\hline $\begin{array}{l}\text { Entrevistas en profundidad a responsables de Cáritas Diocesana e } \\
\text { interparroquiales }\end{array}$ & 4 & 4 & $100 \%$ \\
\hline Cáritas parroquiales representadas en la encuesta al voluntariado & 65 & 40 & $61 \%$ \\
\hline Total de voluntariado de Cáritas encuestado & 840 & 100 & $12 \%$ \\
\hline $\begin{array}{l}\left(^{*}\right) \mathrm{N}=\text { número de elementos del universo poblacional. } \\
\left(^{* *}\right) \mathrm{n}=\text { número de elementos de la muestra. }\end{array}$ & & \\
\hline
\end{tabular}

En total hemos realizado 72 entrevistas, repartidas, como se observa en la tabla, entre informantes privilegiados al inicio del estudio, arciprestes, comunidades religiosas, centros sociales y responsables de las Cáritas interparroquiales y la diocesana. También obtuvimos información a través de encuestas directas a 100 voluntarios de las Cáritas parroquiales, voluntarios que representan aproximadamente un $12 \%$ del total con el que cuenta la entidad en la Archidiócesis.

Somos conscientes de que no hemos abarcado todo el complejo entramado de entidades de acción social de la Iglesia, pero el trabajo de campo refleja una parte importante de lo que se lleva a cabo en el territorio. El voluntariado de las cáritas parroquiales queda menos representado en el total del trabajo de campo, pero hay que tener en cuenta que este colectivo presenta rasgos muy homogéneos, tal y como indican los registros que maneja Cáritas Diocesana. Las 100 entrevistas realizadas resultan, por lo tanto, una buena aproximación a las características del voluntariado de esta entidad.

El trabajo de campo tuvo una duración aproximada de nueve meses, dadas las dificultades de encontrar huecos para llevar a cabo las entrevistas, de modo que se realizaron en un tiempo que para cualquier investigación social podría resultar excesivo. Sin embargo, estamos satisfechos tanto del proceso como de los resultados, ya que nos hemos enfrentado a un objeto de estudio muy peculiar por lo que respecta a su funcionamiento y su organización.

La estructura de la comunicación es la siguiente. En la primera parte, «El modelo organizativo de la acción social», presentamos los rasgos de la organización de las entidades católicas que desarrollan la acción social en el territorio. Nuestro análisis se centra aquí en el análisis y la adecuación de los conceptos utilizados en la sociología de las organizaciones respecto a las características organizativas y de funcionamiento de la Iglesia católica. El resultado es la caracterización de la acción social como un complejo entramado de entidades que funcionan en red a modo de una constelación como forma organizativa. En la segunda parte abordamos la conexión entre la forma organizativa 
de la Iglesia católica y el alcance de la prestación social que lleva a cabo. Es aquí donde argumentamos la eficacia del alcance de esta prestación en función de la descentralización como marca idiosincrásica de la organización de estas entidades de acción social. En la tercera parte, de forma muy resumida, exponemos algunas características de las entidades entrevistadas. Por último, en el apartado de conclusiones sistematizamos los principales hallazgos del estudio realizado.

\section{El modelo organizativo de la acción social}

En sentido general se puede afirmar que toda organización y su funcionamiento son el resultado de la combinación de una serie de elementos internos y externos, o, como se denomina en la teoría de la dirección y en la sociología de las organizaciones, sus recursos organizativos. Las peculiaridades de la Iglesia católica y de sus entidades que podríamos denominar «de base» hacen que su organización y su funcionamiento se asemejen a lo que en la literatura de la sociología de las organizaciones se ha venido reconociendo como las estructuras en forma de constelación, cuya característica fundamental es una configuración basada en la red como forma estructural y en la autonomía organizativa como forma de actuar.

Pues bien, las entidades católicas que llevan a cabo la acción social presentan un tipo de configuración similar al de una constelación. También podemos imaginar la organización de la acción social y sus entidades como una «estructura en explosión estelar», tal como la caracterizan Bueno y Morcillo (1994: 376).

\section{Figura 1. Modelo de organización en constelación}

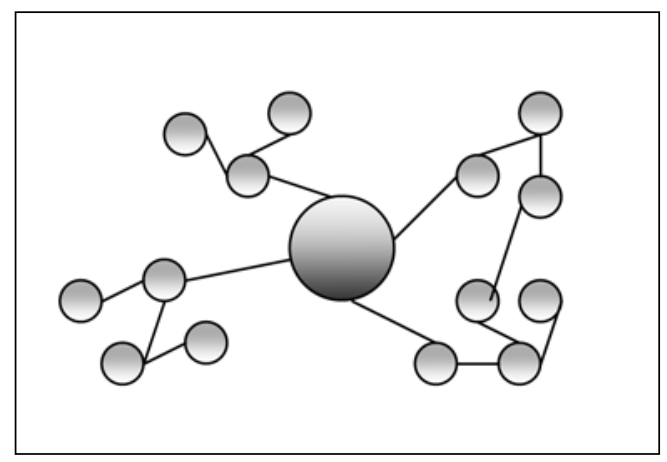

El modelo de la constelación aplicado a las entidades católicas nos lleva a considerar algunas de sus características básicas. Siguiendo los parámetros que propone Schein (1972), las entidades de la Iglesia configuran una organización con una coordinación 
racional de las actividades de personas que intentan conseguir una finalidad común y explícita, mediante la división de las funciones y del trabajo, y a través de una autoridad y de la responsabilidad compartida. Pues bien, la acción social que lleva a cabo la Iglesia entra dentro de los parámetros de esta amplia definición: (1) se trata de un grupo de personas (2) que tienen fines comunes y explícitos, (3) que se reparten las funciones a realizar y (4) que están sometidas a una ordenación de sus acciones en función de algún tipo de autoridad.

En este punto es necesario hacer una apostilla. Obsérvese que se dice explícitamente funciones y/o acciones sometidas a algún tipo de autoridad. En el contexto del estudio que hemos llevado a cabo, no identificamos la autoridad con la jerarquía eclesiástica, es decir, con un tipo de estructura de mando que puede ser más o menos piramidal o clásico. Aquí entendemos por autoridad la acepción de auctoritas, la potestad, la facultad o legitimidad que orienta una determinada acción. Se trata, por lo tanto, de otro tipo de fuente de autoridad que se basa en la propia acción más que en un sistema jerárquico reconocido.

También podemos entender la autoridad como el prestigio y crédito que se reconoce a una persona o institución por su legitimidad o por su calidad y competencia en alguna materia. En este sentido, observamos cómo las entidades que desarrollan la acción social vinculadas a la Iglesia se dotan de esa autoridad para poder funcionar y desarrollar su labor. Se trataría de una especie de autoridad emanada de algún tipo de regla consuetudinaria y de la tradición en que se inscribe la propia acción social de la Iglesia. La acción social que realiza el voluntariado ligado a la Iglesia desarrolla su tarea bajo el principio de legitimidad que la propia Iglesia tiene de manera colectiva y comunitaria: se trata de una interiorización de la tarea común que no necesita el consentimiento de una persona o autoridad por encima de la propia acción. Cada grupo o entidad comparte una autoridad moral que emana de un mismo tronco común. Aquí descubrimos, con efectos prácticos, el primer rasgo de la estructura descentralizada y autónoma de las entidades que llevan a cabo la acción social.

Veamos ahora qué nos dice Zerilli (1988) a partir de la definición anterior, que, formulada de otra manera, introduce un elemento nuevo, no tanto porque no aparezca en la anterior definición, sino por la importancia que adquiere en ésta. Las entidades católicas que realizan acción social compartirían cuatro elementos, a saber, a) la presencia de una finalidad u objetivo común a alcanzar; b) la presencia de un conjunto de personas y de medios técnicos, económicos y de otra naturaleza; c) la combinación de los esfuerzos de las personas y los medios - lo que podemos llamar recursos organizativos - según un esquema oportunamente estudiado que asegure la consecución de los objetivos, y d) un sistema de dependencias y relaciones que lleve a coordinar los esfuerzos hacia la consecución de los objetivos. Si nos fijamos con detenimiento en esta 
definición, Zerilli nos habla de combinación de esfuerzos de un sistema de dependencias de las relaciones que se orientan a la coordinación.

Creemos que aquí radica una de las especificidades más destacadas de la organización de la acción social de la Iglesia. La pregunta es pertinente: ¿qué tipo de coordinación de las actividades de orientación social se produce en el seno de las entidades vinculadas a la Iglesia? La respuesta a esta pregunta nos puede descubrir un tipo de organización con un elevado grado de autonomía organizativa versus una organización en la que las directrices de acción vienen divisionalmente marcadas desde las jerarquías clásicas que corresponden a la estructura formal. El estudio realizado muestra una estructura orientada a lo que en la sociología de las organizaciones se denomina una estructura en red.

El modelo de toma de decisiones que observamos en las entrevistas a las entidades católicas que llevan a cabo acción social coincide con el que propusieron Cohen, March y Olsen (1972). Se trata de un modelo de toma de decisiones propio de organizaciones que tienen que hacer frente a situaciones problematizadas y con una participación que los autores llaman fluida. En este modelo organizacional confluyen simultáneamente problemas, soluciones, decisores y decisiones, y todos estos aspectos interaccionan en una especie de sistema relacional que tiene más que ver con una red (una forma de constelación) que con una organización altamente centralizada.

La Iglesia como organización plantea preguntas interesantes a las definiciones tipológicas ofrecidas por la sociología en torno a la estructura de las organizaciones. $\mathrm{Si}$ consultamos algunas definiciones de la literatura especializada, observaremos cómo la propia idiosincrasia de la Iglesia hace posible un planteamiento de carácter epistemológico.

Una de las definiciones más aceptadas por su grado de generalidad es la que proponen Kast y Rosenzweig (1987). Para estos autores, desde el punto de vista analítico, la organización viene definida por el comportamiento de los individuos que la componen orientado hacia ciertas metas que los miembros del grupo reconocen y conocen de alguna manera. La organización implica actividades estructuradas, es decir, cierto diseño y planificación, y estructuras perdurables y permanentes en la base del comportamiento, que estén integradas, o sea, individuos que trabajan juntos y cooperan en relaciones de interdependencia. La noción de interrelación supone un sistema social. En este sentido, se puede afirmar que las entidades católicas que llevan a cabo acción social consisten en organizaciones (1) orientadas hacia metas (individuos con un propósito) que (2) son sistemas psicosociales (individuos que trabajan en grupo) y (3) tecnológicos (individuos que utilizan conocimientos y técnicas) y (4) se dotan de una integración de actividades estructuradas (individuos que trabajan juntos en relaciones estructurantes). 
Como podemos ver, también esta definición comparte algunos parámetros con anteriores definiciones. Por lo tanto, la Iglesia — en cuanto a su acción social- se puede entender como una organización formada por individuos que actúan movidos por criterios de racionalidad «según finalidades», dentro de un entorno en el que hay una necesaria cooperación para conseguir dichos fines, acompañados de lo que en la teoría de sistemas se denomina sistema tecnológico y que, a efectos de lo que ahora nos ocupa, podemos traducir como el conocimiento teórico-operativo acumulado y bajo un sistema estructurado.

De estas definiciones se pueden derivar algunos elementos que nos ayudan a entender mejor la dinámica organizacional de la acción social de la Iglesia.

El primero de ellos tiene que ver con la estructura social, que implica un modelo de relaciones entre los diferentes actores que participan en la acción social. En un sentido más amplio, a modo de lo que los sistémicos llamarían un subsistema abierto, la estructura social está conformada por varios elementos: a) la estructura normativa externa a la organización, es decir, el contexto legal de acción donde se desarrolla la acción social de la Iglesia; b) la estructura de normas, incluidas las consuetudinarias internas que forman parte del conjunto de reglas de carácter específico para gestionar y/o gobernar el comportamiento de los miembros a fin de lograr las metas previstas, y c) la propia estructura de comportamiento que enfatiza la formalización de los estándares de comportamientos que se exigen a los miembros de la organización.

En segundo lugar, para el análisis que nos ocupa nos puede ser útil considerar, como hace Scott, el elemento de la tipología de los propios actores que participan en la acción social. De entrada, consideramos los miembros que activan y protagonizan esta acción social como movidos por unos intereses particulares y colectivos que influyen de manera relevante en la estructura y en el funcionamiento de la acción social (y en cierto modo, lógicamente también de la propia Iglesia como organización). La tipología del voluntariado que forma el engranaje de la prestación social tiene las siguientes características predominantes: elevada edad, femenino y con nivel de estudios medio.

Seguidamente debemos considerar los propósitos y las finalidades de la propia acción, concebidos aquí como objetivos deseables que los miembros que llevan a cabo la acción social pretenden alcanzar mediante la realización de diversas actividades y su propio trabajo. Una cuestión pertinente a tener en cuenta cuando abordamos este elemento configurador es si se pueden producir conflictos entre los intereses y objetivos que persiguen los diferentes actores a nivel individual o microgrupal, y los intereses y fines que persigue la organización en sus instancias más formales y superiores. El análisis de las entrevistas nos da como resultado una coincidencia muy fuerte en los objetivos o fines que mueven a participar en la acción social, que pueden resumirse en un sentido de evangelización mediante la acción, en un servicio de la caridad. 
Otro elemento constitutivo de la acción organizacional es el que la perspectiva socio-técnica llama tecnología. Ésta se entiende en el sentido de know how, es decir, el conjunto de conocimientos, herramientas, equipos, técnicas, habilidades y destrezas que la organización despliega. Evidentemente, también se contempla aquí el hardward (en sentido figurado) que hace posible todos los tipos de saber que la organización tiene y utiliza. El conocimiento acumulado por el voluntariado de la Iglesia ha dado lugar a un conjunto de rutinas organizativas y una flexibilidad organizativa que nos lleva, de nuevo, a entender esta organización como una red social entretejida alrededor del servicio a los más desvalidos.

Finalmente, el entorno es el último de los elementos a tener en cuenta para el estudio de la acción social; se trata del conjunto de subsistemas —básicamente el económico, el social, el técnico y el cultural- que tienen relevancia a la hora de poner en práctica la acción social en nuestra sociedad. Una idea persistente en las entrevistas en profundidad es que en los últimos años se ha producido un escaso reconocimiento social de la labor que hace la Iglesia por los más desfavorecidos. El contexto social ha infravalorado la labor que llevan a cabo las entidades católicas en favor de los más desfavorecidos de nuestra sociedad. En las entrevistas este tema es recurrente: «no se valora suficientemente la acción que hacemos porque es desconocida», «no tenemos instrumentos para llegar a la gente y decirle lo que hacemos», «no tenemos a nuestro favor los medios de comunicación a la hora de dar a conocer nuestra actividad»... son algunas de las opiniones que han expresado los entrevistados que realizan acción social.

Sin embargo, una de las instituciones clásicas de prestación social como es Cáritas aparece en la «Encuesta de Condiciones de Vida de la Población de Tarragona» ${ }^{1}$ como la más utilizada por las familias que han pedido alguna vez asistencia social, seguida de los servicios municipales. También aparece como la mejor valorada por la sociedad en general.

\section{Sobre la acción social y su alcance}

Gutiérrez (1993: 112) indica en su introducción a la descripción histórica de la presencia de Cáritas en la sociedad española que «el futuro se inscribe ahora en clave diferente: la crisis del Estado del bienestar no es sólo crisis económica: es también crisis axiológica, estética, política y desde los foros internacionales se ve la necesidad de la existencia del mayor número posible de instituciones intermedias que, entre el Estado

\footnotetext{
1 Según datos de la «Encuesta de Condiciones de Vida de la Población de Tarragona» (ECVPT) de 2007, dirigida por A. Belzunegui e I. Brunet, realizada por la URV y patrocinada por Cáritas Interparroquial de Tarragona, la Diputación de Tarragona y el Ayuntamiento de la ciudad. Esta encuesta es una adaptación de la «Encuesta de Condiciones de Vida» del Instituto Nacional de Estadística y recabó información de 931 hogares de la ciudad de Tarragona durante el año 2007 a través de encuesta.
} 
y el ciudadano, satisfagan, en colaboración, necesidades culturales y socio-ambientales (por la falta de comunicación, de espacio, de identidad) y no sólo necesidades materiales».

Las entidades católicas aparecen como organizaciones intermediadoras entre el individuo y la colectividad, además de servir en muchas ocasiones como vínculo también con las instituciones públicas asistenciales.

La actividad socio-asistencial de la Iglesia se vertebra sobre la base de la filosofía de algunos textos fundacionales que recoge Abril (2008), como el de Maillo García en el número 3 de la revista Documentación Social cuando afirma que «hablando con rigor, las actividades asistenciales encaminadas a remediar necesidades y deficiencias humanas, tal y como se entienden hoy, son las mismas obras de beneficencia y de caridad que, desde su fundación, constituyen uno de los distintivos genuinos de la Iglesia católica». Ahora bien, inmediatamente apuesta por una sistematización de la ayuda a los necesitados cuando añade: «la ayuda a los necesitados ha tenido en todas partes un doble carácter: por un lado, ha sido individual; por otro, no ha contado con un plan que gradúe las necesidades y ayudas, adecuando éstas a aquéllas de una manera científica. Pero un plan de ayuda social es algo más que una serie de ficheros donde se registran datos personales de los necesitados y cuantía y fecha de los recursos que se dedican».

Gran parte de esta acción se basa en ir a las fuentes de la realidad de las personas, de la realidad de los grupos y, en los tiempos que corren, también de la realidad de las culturas, ya que buena parte de esta acción social se dirige actualmente a cubrir las necesidades de personas inmigrantes. La atención y el voluntariado que desarrollan los grupos de la Iglesia frecuentemente colocan a sus miembros en las fronteras de la gestión de las situaciones de marginalidad. De hecho, buena parte de esta acción tiene como destinatarias personas que escapan a la atención social de las administraciones por sus condiciones de extrema marginalidad y exclusión social.

Esta atención a los sectores más excluidos de la sociedad no se produce como resultado de una circunstancia al azar, sino que forma parte del resultado de un tipo de organización muy descentralizada que funciona como una malla en el territorio y con una gran flexibilidad en cuanto a la atención directa. Se trata de una situación en la que los grupos de acción social y las personas que los integran responden a lógicas de funcionamiento ad hoc, es decir, un tipo de funcionamiento que trata los problemas cotidianos en función de la proximidad de los propios miembros que ejercen la acción sin tener demasiado en cuenta el contexto organizativo más amplio. Aquí se pone de manifiesto el principio de subsidiariedad al que hacía referencia hace 50 años el Dr. R. Duocastella, director nacional de la Sección Social de Cáritas.

La acción social de la Iglesia se caracteriza por desarrollarse simultáneamente a la de otras instancias que también trabajan en el ámbito de las situaciones de desigualdad 
social. El principio dominante en la mayoría de las entidades estudiadas es, en primer lugar, dar el máximo de servicios y prestaciones a todas aquellas personas que los piden y necesitan, y, en segundo lugar, asegurar el mínimo indispensable de servicios para que esas personas puedan salir de la manera más rápida de su situación.

El estudio de la acción social pone de manifiesto la integración y coordinación entre las entidades y organizaciones que la llevan a cabo, una coordinación que, con todo, puede conllevar en algunos casos cierta duplicidad en el trabajo realizado, sobre todo el más burocrático, como, por ejemplo, el trabajo con las instituciones del territorio. El perfil de los destinatarios, muchas veces con un elevado grado de movilidad, hace que esta situación se dé con bastante facilidad.

Sin embargo, ante la burocratización de los procedimientos que ponen en marcha las diferentes administraciones, las organizaciones ligadas a la Iglesia tienen una actuación mucho más rápida y actúan directamente sobre los problemas de las personas. Éste es un valor añadido que ofrecen estas organizaciones en su acción social. Precisamente el propio funcionamiento autónomo de estas organizaciones las hace más ágiles ante la problemática de la exclusión social. De ahí que sean las diferentes Cáritas las entidades que más trabajan a nivel comunitario y las más buscadas por los propios beneficiarios últimos de las prestaciones que se dan.

\section{Tabla 2. Entidades e instituciones a las que recurren las familias con dificultades}

\begin{tabular}{|l|c|c|}
\hline & $n$ & $\%$ \\
\hline Servicios sociales municipales & 93 & 10 \\
\hline Consejo Comarcal & 35 & 3,8 \\
\hline Generalitat de Cataluña & 72 & 7,7 \\
\hline Servicios del Estado & 36 & 3,9 \\
\hline Cruz Roja & 53 & 5,7 \\
\hline Cáritas & 148 & 15,9 \\
\hline Otros & 6 & 0,6 \\
\hline Total de hogares de referencia & 931 & - \\
\hline
\end{tabular}

Fuente: «Encuesta de Condiciones de Vida de la Población de Tarragona», 2007.

Como se observa en la tabla anterior, la entidad a la que más recurren las familias con dificultades es Cáritas, con un $15,9 \%$ de hogares que han acudido alguna vez a pedir algún tipo de ayuda. Le siguen en importancia los servicios municipales de bienestar social, con un $10 \%$ de hogares, y los servicios de la Generalitat de Cataluña, con un $7,7 \%$. 
La entidad más conocida en cuanto a su actividad es Cáritas (el 57,8\% de los individuos entrevistados en la «Encuesta de Condiciones de Vida de la Población de Tarragona»), seguida de la Cruz Roja (el 55,6\%).

Tabla 3. Conocimiento de las diferentes entidades e instituciones que realizan acción social

\begin{tabular}{|l|c|c|c|}
\hline & No conoce & Sí conoce & \\
\hline Cáritas & $\% \%$ & $\%$ & Total \\
\hline Cruz Roja & 42,2 & 57,8 & 100 \\
\hline ONG & 44,4 & 55,6 & 100 \\
\hline Servicios sociales del Ayuntamiento & 57,0 & 43,0 & 100 \\
\hline Servicios sociales de la Generalitat & 59,4 & 40,6 & 100 \\
\hline Servicios sociales del Estado & 68,2 & 31,8 & 100 \\
\hline Servicios sociales del Consejo Comarcal & 69,1 & 30,9 & 100 \\
\hline
\end{tabular}

Fuente: «Encuesta de Condiciones de Vida de la Población de Tarragona», 2007.

El mayor desconocimiento de los entrevistados tiene que ver con la tarea que realizan los servicios sociales del Consejo Comarcal (el 77,7\%). Le siguen, en desconocimiento, el 69,1\% que desconoce la labor que llevan a cabo los servicios sociales del Estado, seguido del $68,2 \%$ que no conoce la actividad de los servicios sociales de la Generalitat y el $59,4 \%$ que no conoce la labor que realizan los servicios sociales del Ayuntamiento.

En la ECVPT se preguntó también sobre la valoración de los ciudadanos de las diferentes entidades e instituciones que llevan a cabo acción social. Las mejor valoradas son la Cruz Roja, con un 63,7\% de entrevistados que valoran su acción como positiva o muy positiva, seguida de las ONG, con un 55\%, y de Cáritas, con un 53,5\%.

Tabla 4. Valoración de la labor realizada por las entidades e instituciones

\begin{tabular}{|l|c|c|c|}
\hline & $\begin{array}{c}\text { Negativa/muy } \\
\text { negativa }\end{array}$ & Indiferente & Positiva/muy positiva \\
\hline Cáritas & $\%$ & $\%$ & $\%$ \\
\hline Cruz Roja & 17 & 29,5 & 53,5 \\
\hline ONG & 9,4 & 26,9 & 63,7 \\
\hline Servicios sociales del Ayuntamiento & 24,3 & 20,7 & 55 \\
\hline Servicios sociales de la Generalitat & 35,6 & 28,9 & 35,6 \\
\hline Servicios sociales del Estado & 32,4 & 28,3 & 39,3 \\
\hline $\begin{array}{l}\text { comarcal Servicios sociales del Consejo } \\
\text { Comarcal }\end{array}$ & 39,7 & 31,2 & 29,1 \\
\hline
\end{tabular}

Fuente: «Encuesta de Condiciones de Vida de la Población de Tarragona», 2007. 
Tenemos también información de la valoración que hacen los hogares pobres y que recurren a la asistencia social de las entidades e instituciones que se la facilitan. Los resultados están expresados en el siguiente gráfico:

\section{Gráfico 1. Valoración positiva/muy positiva de la acción de las entidades según los hogares pobres}

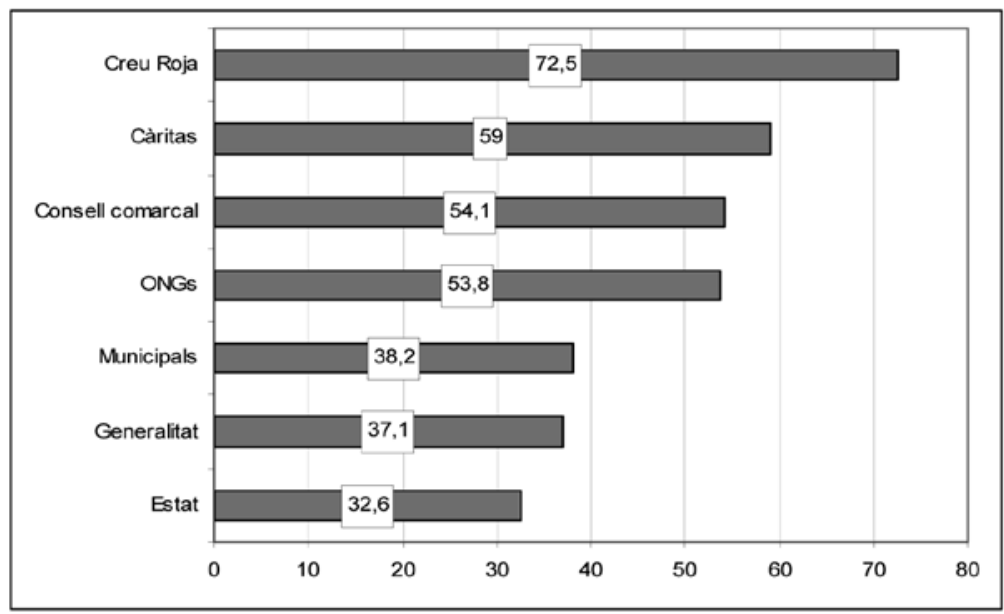

Fuente: «Encuesta de Condiciones de Vida de la Población de Tarragona», 2007.

La Cruz Roja es la mejor valorada por los hogares pobres de la ciudad, pues un $72,5 \%$ de ellos considera su actuación como positiva o muy positiva. Le siguen Cáritas, con un $59 \%$ de valoración positiva, el Consejo Comarcal $(54,1 \%)$, las organizaciones no gubernamentales $(53,8 \%)$, los servicios municipales $(38,2 \%)$, los servicios de la Generalitat $(37,1 \%)$ y, por último, los servicios del Estado $(32,6 \%)$.

A diferencia también en ocasiones de las instancias administrativas y de la profesionalización de la asistencia social, la acción social de la Iglesia trabaja desde un concepto integral de la persona, es decir, no reduce la persona ni el concepto de necesidad a una sola escala, sino que tiene una visión más integral de la promoción social y del desarrollo comunitario. Este hecho también es reconocido por los propios entrevistados como un valor añadido de la acción social. El tratamiento integral de la persona se ha convertido, por lo tanto, en un signo distintivo de la acción social de la Iglesia. Esta idea queda patente también en uno de los textos fundacionales sobre la actividad caritativa de los católicos: «Un Estado que se preocupa honradamente del bienestar de los pobres: crea organismos para aliviar las diversas necesidades, no pone límite al número de funcionarios, retribuyéndoles dignamente; distribuye títulos que acreditan su competencia; organiza una red burocrática de admirable buen sentido..., pero, al elegir 
el personal que da vida a esta maquinaria filantrópica, desestima los institutos religiosos... prefiriendo a empleados que se acogen a estas plazas sin vocación y cumplen su tarea con la mecánica fidelidad del oficial que lo es para cobrar un sueldo de plantilla. El pobre recibirá ayuda, sí, pero con la misma prosaica indiferencia con la que se llena un padrón y se pega una póliza» (Gutiérrez, 1993: 321).

Si tenemos en cuenta la opinión de los entrevistados acerca de su trabajo de acción social y la percepción que mayoritariamente tienen sobre su escasa consideración y reconocimiento sociales, se podría decir que es, en cierto modo, un trabajo invisible. Utilizamos aquí el paralelismo con el trabajo doméstico que realizan la mayoría de las mujeres en nuestra sociedad. Es bien sabido que gran parte de este trabajo que llevan a cabo las mujeres permanece socialmente invisible para la mayoría de la sociedad. Ellas, a diferencia de los hombres, se encargan de la mayoría de tareas que tienen que ver con el cuidado de las personas, en el seno de la familia y atendiendo a personas en situaciones de dependencia. Pues bien, no es tampoco casual que la mayoría del voluntariado que trabaja en y para las organizaciones sociales de la Iglesia sean mujeres. Sobre ellas recae gran parte del trabajo que asegura el bienestar social, físico y emocional de las personas en nuestras sociedades. Tanto a nivel de la familia como de las entidades que acogen y acompañan a las personas con dificultades para poder insertarse en los contextos sociales.

Hemos hablado antes del valor añadido que supone el reconocimiento inmediato de las situaciones de dificultades sociales y vivenciales de las personas que acuden a las organizaciones de la Iglesia. Decíamos que el hecho de poder responder con inmediatez a estas demandas ya supone un plus respecto a otras instituciones que tienen procedimientos burocráticos más complejos. Pero se nos plantea aquí otro interrogante: ¿hasta qué punto el trabajo de las personas de estas organizaciones hace posible que los individuos demandantes de prestaciones puedan desarrollar trayectorias vitales autónomas e insertadas en nuestra sociedad? De hecho, es un interrogante que puede plantearse a nivel general a todas las entidades e instituciones que se dedican a las situaciones de riesgo de exclusión.

En términos porcentuales y según nuestros interlocutores, entre un 50\% y un 75\% de los individuos que reclaman algún tipo de ayuda o de prestación social no puntual desarrollan una trayectoria positiva en cuanto a su inserción social. Esto se comprueba a través del seguimiento que hacen estas mismas organizaciones. En los últimos años, el mayor grado de éxito se da entre las personas y las familias inmigrantes.

Buena parte de la acción social está vinculada a familias inmigrantes. Vienen con necesidades económicas y afectivas, y las organizaciones de la Iglesia son unas de las primeras con las que contactan. Podríamos decir que en la actualidad hay dos instituciones cruciales a la hora de llevar a cabo la acogida de estas familias: una es la 
institución escolar y la otra es la religiosa. Las administraciones aparecen cuando las familias ya han entrado en contacto con ambas. El grado de eficacia de las tres entidades depende de muchos factores, como, por ejemplo, del grado de interconexión que se establece entre las tres instituciones en relación con la acogida y con la prestación social, así como con el seguimiento de la problemática específica.

\section{La acción social en cifras}

\subsection{Las comunidades religiosas}

En el estudio contestaron el cuestionario un total de 43 comunidades religiosas de 34 órdenes religiosas diferentes. Se obtuvo un $72 \%$ de respuestas del total de comunidades que llevan a cabo acción social en el territorio.

El quehacer de las congregaciones en general y de las comunidades en particular abarca un amplio abanico de actividades que van desde las más contemplativas, centradas fundamentalmente en la oración y que, a efectos de la recogida de información, se desestimaron, hasta las más prácticas en el sentido de ocuparse de las personas que presentan necesidades de tipo material, físico, psíquico o emocional.

Las actividades que pueden considerarse como más claramente incardinadas dentro del ámbito de la acción social de la Iglesia vienen enmarcadas por una misión en la vida de las personas que se puede identificar como «hacer el bien al prójimo». Esta formulación e intención genéricas recorren todos los discursos de los entrevistados; en última instancia, como dice Soro (2008: 199), se trata de «que el sujeto de la acción caritativa es la Iglesia toda».

Los entrevistados también reclaman una acción directa en contacto con los problemas del conjunto de la sociedad, y, en particular, de aquellos que más la necesitan. De lo que se trata también es de «realizar un ejercicio empático»; «(...) difícilmente comprenderemos la situación y encontraremos soluciones a los problemas de los pobres si vivimos excesivamente alejados de su situación», «la proximidad es imprescindible para una buena intervención y, sobre todo, para una aproximación cordial» (Soro, 2007: 203). Aquí se vuelve a poner de manifiesto el principio de subsidiariedad con el que se mueven las entidades católicas en la prestación de asistencia social: la proximidad alcanza entonces un plus en la tarea a realizar ya que posibilita la inmediatez de la prestación.

Otra cuestión de interés es ver a qué colectivos dirigen primordialmente la acción social las comunidades y congregaciones. Más de la mitad de las comunidades dirigen sus esfuerzos de acción social y asistencial a las personas de la tercera edad $(53,5 \%)$ y a las familias y menores $(51,2 \%)$. 
Tabla 5. Colectivos a los que se dirige la acción social de las comunidades

\begin{tabular}{|l|c|}
\hline & $\%$ \\
\hline Tercera edad & 53,5 \\
\hline Menores y familia & 51,2 \\
\hline Enfermos & 48,8 \\
\hline Jóvenes & 42,0 \\
\hline Inmigrantes & 37,2 \\
\hline Discapacitados & 25,6 \\
\hline Mujeres & 23,3 \\
\hline Sin techo & 16,3 \\
\hline Drogodependientes & 11,6 \\
\hline Reclusos & 11,6 \\
\hline Sanidad & 9,3 \\
\hline Minorías étnicas & 7,0 \\
\hline Parados & 7,0 \\
\hline
\end{tabular}

Fuente: «Encuesta sobre la Acción Social de la Iglesia», 2008.

La atención a las personas de la tercera edad, primer colectivo en cuanto a intensidad de la atención, se dirige a personas que viven solas, gran parte de ellas mujeres con escasos recursos y/o con problemas de dependencia. Buena parte de esta atención se desarrolla en los centros para la tercera edad que gestionan las comunidades religiosas de forma privada y/o concertada con las administraciones.

Tabla 6. Colectivos a los que se dirige la acción social según el tipo de comunidad. Porcentajes $\left({ }^{*}\right)$

\begin{tabular}{|l|c|c|c|}
\hline & Total & Comunidades femeninas & Comunidades masculinas \\
\hline & $\%$ & $\%$ & $\%$ \\
\hline Tercera Edad & 53,5 & 58,8 & 33,3 \\
\hline Menores y familia & 51,2 & 50,0 & 55,6 \\
\hline Enfermos & 48,8 & 55,9 & 22,2 \\
\hline Jóvenes & 42 & 38,2 & 55,6 \\
\hline Inmigrantes & 37,2 & 35,3 & 44,4 \\
\hline Discapacitados & 25,6 & 26,5 & 22,2 \\
\hline Mujeres & 23,3 & 23,5 & 22,2 \\
\hline Sin techo & 16,3 & 8,8 & 44,4 \\
\hline Drogodependientes & 11,6 & 8,8 & 22,2 \\
\hline Reclusos & $11,611,6$ & 8,8 & 22,2 \\
\hline Sanidad & 9,3 & 8,8 & 11,1 \\
\hline Minorías étnicas & 7 & 2,9 & 22,2 \\
\hline Parados & 7 & 2,9 & 22,2 \\
\hline$\left(^{*}\right.$ Los porcentajes señalan múltiples respuestas, por lo que no suman el 100\%. \\
\hline
\end{tabular}

Fuente: «Encuesta sobre la Acción Social de la Iglesia», 2008. 
Las comunidades femeninas se encargan, además, de las problemáticas asociadas a las personas con mayor grado de dependencia funcional y/o psíquica, bien sean personas mayores o discapacitadas en general. Muchas veces estas problemáticas de dependencia tienen que ver con procesos crónicos de enfermedades.

Las comunidades masculinas se encargan de las formas más marginales de la exclusión social, como drogodependientes, reclusos, personas sin techo o vagabundos. Son colectivos que presentan una intensidad fuerte en cuanto a pobreza, privación y vulnerabilidad social.

Otra cuestión a tratar es el perfil del voluntariado con que cuentan las comunidades religiosas para llevar a cabo su labor social y asistencial en la comunidad. La media de voluntarios que participan en las 43 comunidades es de ocho. El 50\% de las comunidades cuentan con el apoyo de cuatro personas como máximo. Si agrupamos las comunidades según el número de voluntarios que las ayudan en su tarea cotidiana de acción social, vemos que el $58,1 \%$ cuenta con la presencia de entre una y cinco personas. El $23,3 \%$, tiene entre seis y diez voluntarios y el 16,3\% tiene más de diez voluntarios.

\subsection{Centros sociales}

Hemos recogido información de 10 centros asistenciales gestionados por entidades católicas, sobre un total de 12, para conocer el alcance de su acción social. Por lo tanto, hemos recogido información del $83 \%$ de los centros asistenciales.

Se puede afirmar que los centros asistenciales ligados a la Iglesia tienen una importante presencia territorial. Se trata de la entidad privada con mayor presencia en cuanto a la realización de acción social, bien sea por iniciativa propia, bien en colaboración con otras entidades y/o instituciones.

La mayor parte de la acción que se lleva a cabo en estos centros no se aborda desde una perspectiva exclusivamente individual, sino que se realiza desde el punto de vista de la intervención comunitaria. En este sentido, este tipo de tarea se acerca mucho más al perfil del trabajo social comunitario que al perfil del trabajo de beneficencia.

Los colectivos beneficiarios de la acción social de los centros son variados. El 50\% de los centros presta servicios relacionados con las problemáticas de la familia y de los menores. El 30\% se ocupa de cuidar a los marginados sin hogar, los vagabundos y los transeúntes. Un centro se dedica fundamentalmente a ayudar a los drogodependientes. El 20\% trabaja también con problemáticas asociadas a los jóvenes, así como también a las mujeres.

En general, los centros se han especializado en determinados colectivos así como en determinados servicios destinados a ellos. Se puede hablar de una cierta profesionalización de los servicios, aunque en muchos casos parte de su actividad sea llevada a cabo por voluntariado. 
Entre los servicios que prestan los centros vinculados a la Iglesia se pueden destacar: la atención a personas con deficiencias psíquicas y sensoriales; los programas de rehabilitación, educación y atención a la discapacidad física y psíquica; el tratamiento integral de personas drogodependientes, su seguimiento sanitario y los programas formativos y de inserción laboral; los comedores sociales; el alojamiento para transeúntes; el asesoramiento a reclusos y ex reclusos; los programas de atención y orientación familiar; los programas específicos de atención a la infancia; los programas formativos $y$ de inserción laboral para jóvenes; el acogimiento de personas mayores y su cuidado, y la atención y el seguimiento de inmigrantes, especialmente a través de programas de formación e inserción sociolaboral.

Los 10 centros han firmado en total 18 convenios con diferentes instituciones. La Administración autonómica es la que mayor número de convenios ha firmado con los centros; en concreto, el $50 \%$ de los convenios se han establecido con diferentes consejerías de la Generalitat de Cataluña. El 28\% de los convenios se han firmado en colaboración con el ayuntamiento donde se ubica el centro. El Gobierno español también participa en un $11 \%$ de los convenios. Los consejos comarcales y las entidades privadas participan en el 5,6\% de los convenios totales.

Los centros cuentan con un total de 68 colaboradores, el $84 \%$ de los cuales son mujeres y el 16\%, hombres. El 63\% son seglares y el 37\%, religiosos. El 51,5\% de los colaboradores de los centros son mujeres seglares y el 32,4\%, mujeres religiosas. Comparativamente, la presencia masculina es bastante menor, ya que hay un $12 \%$ de hombres seglares y un 4,4\% de religiosos.

En los 10 centros entrevistados hay un total de 78 personas vinculadas a ellos por contrato. La mayoría del personal empleado tiene un nivel de estudios secundarios, concretamente el 56,4\%. El 30,8\% del personal tiene título universitario. Entre estas dos categorías suman el 87,2\% de los empleados. El 12,9\% restante se reparte entre los que tienen estudios de graduado escolar y los que tienen estudios primarios sin título. El tipo de actividades que lleva a cabo el personal empleado en los centros sociales exige un determinado grado de cualificación, como, por ejemplo, tareas relacionadas con los servicios sanitarios, tareas formativas y educativas, administración y gestión de centros, etc.

Respecto a la edad, los menores de 30 años representan el 17\% de los empleados de los centros de la Iglesia. El grupo de edad más numeroso es el comprendido entre los 41 y los 50 años, concretamente un $37 \%$ de los empleados están dentro de esta franja de edad.

Por último, el voluntariado está presente en el $80 \%$ de los centros de la Iglesia. El número total de voluntarios y voluntarias que participan en los centros es de 243 personas. Siguiendo el patrón del voluntariado que participa en las actividades de las 
comunidades religiosas (recordemos que entre el voluntariado de las comunidades religiosas hay un $80 \%$ de mujeres y un $20 \%$ de hombres), aquí también predominan las mujeres: un $73 \%$ del voluntariado son mujeres y un $27 \%$, hombres.

Respecto a su nivel de estudios, hay una menor presencia de personas con titulación universitaria (un 15\%) en favor del voluntariado con estudios secundarios, que representa el $32 \%$ del total, o con estudios primarios (un 28\%). Uno de cada cuatro voluntarios tiene el graduado escolar.

El grupo de edad mayoritario entre el voluntariado que presta sus servicios en los centros es el comprendido entre los 41 y los 65 años, concretamente el 51,5\% de los voluntarios. Uno de cada cinco voluntarios tiene menos de 30 años $(19,7 \%)$ y el $28,4 \%$ tiene hasta 40 años.

\subsection{La acción social de Cáritas}

Las Cáritas están repartidas por prácticamente todas las parroquias de la Archidiócesis de Tarragona y prestan servicios sociales a las personas más necesitadas, con un grado de autonomía importante unas de otras, así como también de las interparroquiales y de la diocesana, las estructuras que las agrupan. Probablemente el modelo organizativo de los grupos de Cáritas es el más parecido al modelo de constelación al que venimos haciendo referencia.

Las Cáritas funcionan con el trabajo voluntario de las personas que se vinculan a ellas. Su financiación proviene, fundamentalmente, de la obtención de dinero en campañas y de donaciones de personas y entidades. También existe una vía de financiación a través de subvenciones a programas concretos de actuación en el ámbito de la protección social, pero el destino de estas subvenciones es cubrir los gastos derivados de la puesta en marcha de dichos programas.

Es difícil precisar el número total de voluntarios y voluntarias adscritos a las Cáritas parroquiales de la unidad territorial en la que se ha realizado el estudio. Según datos del registro de Cáritas Diocesana, el voluntariado asciende a unas 840 personas aproximadamente. El perfil del voluntario de las Cáritas parroquiales es el de una mujer de más de 50 años y con estudios primarios y/o secundarios que realiza tareas muy variadas de atención a los solicitantes de ayuda o a las personas de su entorno que están identificadas como personas más vulnerables social y económicamente hablando. $\mathrm{Al}$ igual que en el caso del voluntariado de las comunidades religiosas y de los centros de la Iglesia, la mayoría de los voluntarios de las Cáritas parroquiales de la Archidiócesis son mujeres. En concreto, tres de cada cuatro voluntarios.

Tal como se ve, las mujeres son las que más se implican en las tareas de voluntariado de las diferentes entidades de la Iglesia. Podemos decir que asistimos a una feminiza- 
ción del voluntariado. Las trayectorias vitales de las mujeres conllevan que estén más vinculadas a las tareas de atención, cuidado y bienestar de las personas. Esta mayor presencia de mujeres en el voluntariado es el reflejo de esa mayor vinculación.

En comparación con otros grupos de voluntariado de las entidades religiosas, el voluntariado de los centros muestra una mayor presencia de personas de más de 65 años. La media de edad es de 64,2 años. No hay diferencias entre la edad media de los hombres voluntarios y la de las mujeres voluntarias: aquéllos presentan una edad media de 62 años y éstas, de 65 años. Las comunidades cuentan con el voluntariado más joven de entre los tres tipos de entidades.

\section{Gráfico 2. Distribución de las edades de los voluntarios de las entidades de la Iglesia}

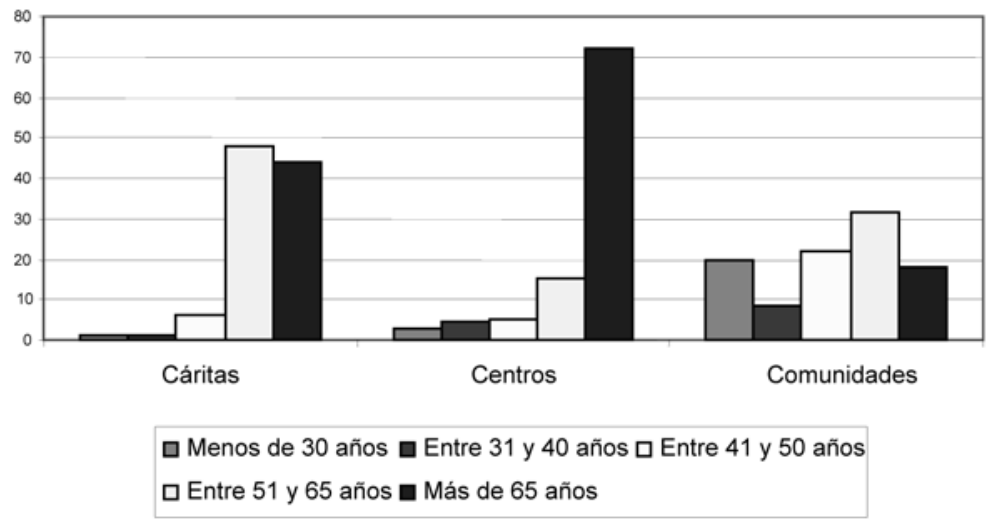

Fuente: «Encuesta sobre la Acción Social de la Iglesia 2008».

Respecto al nivel de estudios del voluntariado de los tres tipos de entidades, los voluntarios que tienen hasta estudios primarios son más numerosos en los centros, un $53 \%$ frente al $46 \%$ de los de Cáritas y el $23 \%$ de los que prestan servicios en las comunidades.

Tabla 7. Estudios del voluntariado de las entidades de la Iglesia

\begin{tabular}{|l|c|c|c|}
\hline & Cáritas & Centros & Comunidades \\
\hline Sin estudios & $2 \%$ & $28 \%$ & $17 \%$ \\
\hline Estudios primarios & $44 \%$ & $25 \%$ & $6 \%$ \\
\hline Estudios secundarios & $40 \%$ & $32 \%$ & $35 \%$ \\
\hline Estudios superiores & $14 \%$ & $15 \%$ & $41 \%$ \\
\hline
\end{tabular}

Fuente: «Encuesta sobre la Acción Social de la Iglesia 2008». 
Los voluntarios con mayor nivel de estudios los encontramos en las comunidades (41\%) y después en los centros (15\%) y en Cáritas (14\%). Cáritas es donde mayor presencia tiene el voluntariado con estudios secundarios, un 40\%, seguido del voluntariado de las comunidades (35\%) y del de los centros (32\%).

\section{Conclusiones}

La acción social de la Iglesia presenta la imagen de una red con diferentes nódulos con mayor o menor centralidad. La forma organizativa que presenta la acción social se asemeja a la de una constelación donde los diferentes grupos que participan lo hacen con una misión común y con un elevado grado de autonomía en su funcionamiento. Las entidades que juegan un papel de cierta centralidad en la red desempeñan labores de coordinación de las tareas que llevan a cabo el resto de entidades, que trabajan con un elevado grado de autonomía. Las entidades con mayor centralidad coordinan más que dirigen los movimientos en la red.

¿Por qué hablamos de constelación como modelo organizativo? Cuando hablamos de las organizaciones vinculadas a la Iglesia católica que se dedican a la acción social, debemos entender una realidad plural y dinámica y con un grado de autonomía elevado. La configuración en constelación se caracteriza por ser una malla o red de relaciones con diferentes nódulos en los que se basan las acciones a desarrollar.

Las relaciones que se establecen en la base de operaciones, es decir, entre los grupos que llevan a cabo acción social, son primordialmente relaciones horizontales en las que predomina la confianza, por el hecho de compartir una misma misión. La comunicación entre los grupos no pasa necesariamente por un órgano central, aunque todos los grupos de acción social informan anualmente de sus actividades; de ahí que los nódulos con mayor centralidad estén ocupados por entidades que desempeñan un papel de coordinación de actividades.

Esta configuración organizativa descansa sobre una filosofía práctica que prioriza la atención social y se resume en la reflexión de una de las personas entrevistadas: «Tenemos que vigilar no convertirnos en instituciones que tienen enormes costes burocráticos, lo que impide que se optimice la llegada de los recursos a los beneficiarios».

Paralelamente, las microorganizaciones que realizan acción social dentro de la Iglesia también pueden concebirse desde el punto de vista organizativo como grupos que se plantean la resolución de problemas ad hoc mediante el uso de los recursos que tienen a su alcance. Gran parte de la acción social llevada a cabo responde a la idea de la primacía de la misma acción sobre la reflexión, lo que supone dar respuestas a medida que van surgiendo las diversas problemáticas. Esto no significa que no exista una determinada sistematización en los procedimientos de actuación. Por lo tanto, estamos ante 
una manera de proceder en que se combinan protocolos de actuación con respuestas ad hoc ante las situaciones particulares.

Estos rasgos de las entidades y de la acción social presentan otra característica que hay que considerar. Las entidades pueden prestar sus servicios de manera ágil, rápida y nada burocrática. En la acción social prima la situación global de la persona que pide ayuda sobre los procedimientos administrativos, que pueden caer en el ritualismo burocrático.

Las entidades de la Iglesia católica ofrecen hoy en día servicios de atención a las personas más excluidas del sistema social. Esto tiene efectos importantes en el engranaje de la prestación social que dan las diferentes instituciones y entidades en el territorio. Tal como se observa en la «Encuesta de Condiciones de Vida de la Población de Tarragona» (ECVPT), en el ámbito de la asistencia social, la entidad más reclamada es Cáritas y también la mejor valorada por el conjunto de la sociedad. Por otra parte, buena parte de la acción social de las entidades se centra en la prestación de diferentes servicios a los colectivos con más riesgo o con mayor vulnerabilidad económica y social. Por poner un ejemplo, el año pasado acudieron a los diversos programas sociales de Cáritas cerca de 40.000 personas en el territorio de la Archidiócesis. El 90\% de los excluidos severos son hoy día atendidos por entidades de la Iglesia católica.

La Iglesia católica ofrece actualmente servicios asistenciales de todo tipo a la mayoría de la población excluida del sistema social. Se presenta como la única institución que garantiza un mínimo de atención a las personas más desposeídas, debido a que las administraciones públicas llegan hasta un nivel de pobreza que podríamos denominar institucional. Los marginados y excluidos también de las administraciones encuentran apoyo en las entidades caritativas de las iglesias. Esta atención está relacionada con la forma organizativa de las entidades analizadas. Comprobamos que, a mayor descentralización y mayor autonomía de actuación, mayor es la cobertura de alcance de las entidades en su conjunto. Por esta razón, estas entidades llegan allá donde no pueden llegar otro tipo de instituciones cuyo modo de proceder está más burocratizado.

Allí donde no llega la prestación social de las diferentes administraciones públicas, las entidades católicas desarrollan su labor de apoyo, acompañamiento y asistencia social. No se trata simplemente de un tipo de asistencia de beneficencia, sino de una asistencia social que quiere responder a las situaciones de injusticia social. Los voluntarios de la Iglesia hablan de una situación de desigualdad social provocada por diferentes factores estructurales que configuran nuestra sociedad. Su discurso está, en todo momento, enmarcado en la existencia de privaciones derivadas del desigual reparto de los recursos económicos y sociales. No se trata de gente desinformada y alejada de la realidad, sino de personas que creen que con su labor individual y humanitaria pueden ayudar a mejorar las condiciones materiales de otras. 
En este sentido, no encontramos diferencias entre los seglares y los religiosos. Si bien estos últimos subrayan la misión pastoral en su discurso sobre la acción social, ambos colectivos coinciden en que la ayuda al prójimo es el primer objetivo del quehacer del cristiano, pues la práctica diaria de todos ellos viene guiada por la misión evangelizadora. Las comunidades religiosas están más estructuradas en torno a sus órdenes, aunque se da un grado de autonomía importante en el funcionamiento del día a día. Las entidades gestionadas por seglares, como, por ejemplo, las Cáritas parroquiales, tienen un funcionamiento autónomo. Conservan el principio de la acción directa como principio fundacional y guía de su acción. Lo importante es actuar y dar respuesta inmediata a las necesidades de las personas desposeídas.

La Iglesia católica presenta un organigrama jerárquico que se difumina en el desarrollo de la acción social. Se trata de una estructura de funcionamiento en la que los diferentes grupos de acción social llevan a cabo sus tareas en consonancia con una idea central de ayuda al prójimo y con una misión personal de proporcionar una vida digna a las personas. Uno de los valores añadidos de la acción social que realizan las entidades de la Iglesia reside, precisamente, en la concepción integral de la persona a la hora de abordar su problemática. La evolución de la acción social ha pasado de una concepción basada en la beneficencia a una actuación más centrada en facultar a las personas para que puedan construir o reconstruir sus trayectorias vitales.

\section{Bibliografía}

Albiol, E. (1960). La Caridad. Madrid: Euroamérica.

Bueno, E. y Morcilllo, P. (1994). Fundamentos de economía y organización industrial. Madrid: McGraw-Hill.

Cohen, M.; March, J. G. y Olsen, J. P. (1972). «Garbage-can model of organizational choice». Administrative Science Quarterly, 17 (1): 1-25.

Consell Pontifici de la Justícia i de la Pau (2005). Compendi de la Doctrina Social de l'Església. Barcelona: Editorial Claret.

Duocastella, R. (1958). «Necesidad de una 'Acción Social' en las Cáritas Diocesanas», recogido en Ana Abril Fernández (2008). «De la acción benéfica a la acción social». Revista Documentación Social, núm. 149-150: 13-30.

Gutiérrez Resa, A. (1993). Cáritas Española en la sociedad del bienestar 1942-1990. Barcelona: Hacer Editorial.

Kast, F. E. y Rosenzweig, J. E. (1987). Administración en las organizaciones: enfoque de sistemas y de contingencias. México: McGraw-Hill. 
Maillo García, A. (1958). «La Asistencia Social y las Escuelas de Servicio Social en España», recogido en Ana Abril Fernández (2008). «De la acción benéfica a la acción social». Revista Documentación Social, n 149-150: 13-30.

Morales Gutiérrez, A. C. (1994). «Diez formas de concebir las organizaciones: una valoración». Revista de Fomento Social, 193 (enero-marzo): 23-65.

Schein, E. H. (1972). Psicología de las organizaciones. Madrid: Prentice-Hall Internacional.

Scotт, W. R. (1992). Organizations: racional, natural and open Systems. Homewood: Prentice-Hall.

Soro Roca, S. (2007). «Los agentes de la caridad». Corintios XIII, 123 (julio-septiembre): 185-205.

Weber, M. (1964). Economía y Sociedad. México: Fondo de Cultura Económico.

Zerilli, A. (1988). Fundamentos de organización y dirección general. Bilbao: Deusto Ediciones. 